\title{
From metamaterials to metasurfaces and metadevices
}

\author{
Yu. S. Kivshar ${ }^{1,2}$ \\ ${ }^{1}$ Nonlinear Physics Center, Australian National University, \\ Canberra ACT 0200, Australia \\ ${ }^{2}$ ITMO University, St. Petersburg 197101, Russia \\ Yuri.Kivshar@anu.edu.au
}

PACS 81.05.Xj, 78.67.Pt, 73.20.Mf

DOI 10.17586/2220-8054-2015-6-3-346-352

\begin{abstract}
Metamaterials are artificial electromagnetic media that are structured on the subwavelength scale. Such structures were initially suggested for achieving the negative index of refraction, but later they became a paradigm for engineering electromagnetic space and controlling propagation of waves through the transformation optics and optically-induced magnetic response. The research agenda is now shifting towards tunable, switchable, nonlinear and sensing functionalities of metamaterials and their applications, and it involves the fields of metasurfaces and metadevices, with the recent demonstrations of breakthrough results with light-controlled metamaterials, nonlinear metamaterials, and tunable metasurfaces for MRI applications. Here, we briefly discuss the basic concepts of this rapidly growing research field, highlighting the recent developments in the physics of metamaterials, subwavelength nanophotonics, and graphene-based photonics.
\end{abstract}

Keywords: metamaterial, metasurface, metadevices, optical magnetism, graphene.

Received: 15 March 2015

Revised: 18 March 2015

\section{What are metamaterials?}

It is well known that the response of any material to applied electromagnetic radiation can be characterized by two electromagnetic parameters, magnetic permeability and electric permittivity. These two physical characteristics are combined in a product to define the square of a refractive index, which measures how fast the material transmits light and how light is bent on entering the material - the higher the refractive index, the slower the propagation and the stronger the deflection. However, a material whose permeability and permittivity become simultaneously negative, termed as a left-handed material, would also allow the propagation of electromagnetic waves with many unusual properties [1-5].

In a left-handed material, the Poynting vector of a wave is anti-parallel to the wave vector and, therefore, the basic feature of light is reversed; that is, light wave propagates in the opposite direction to the energy flow. This leads to some very interesting effects such as the reversal of the Doppler shift for radiation, and the reversal of Cherenkov radiation. In addition, one of the most basic principles of optics, Snell's law, is 'reversed' at the interface of a left-handed medium with a normal right-handed material, so that the electromagnetic waves experience negative refraction, and this property can be employed for flat-lens focusing $[1,4]$.

The creation of novel composite materials demonstrating the left-handed properties at telecommunication and visible frequencies required the development of novel concepts and novel nanofabrication techniques. Many theoretical concepts that have been suggested so far are based around nanofabricated composites. One example is metal nanowires and nanowire plasmonic materials, where nanowires arranged into parallel pairs can act as a left-handed material with the effective magnetic permeability and dielectric permittivity that are both negative in the visible 
and near-infrared spectral ranges [6]. Various composites, based on metallic and dielectric nanostructures that have macroscopic negative refraction and act as left-handed materials, have recently been suggested and developed [7-10] (see Fig. 1).
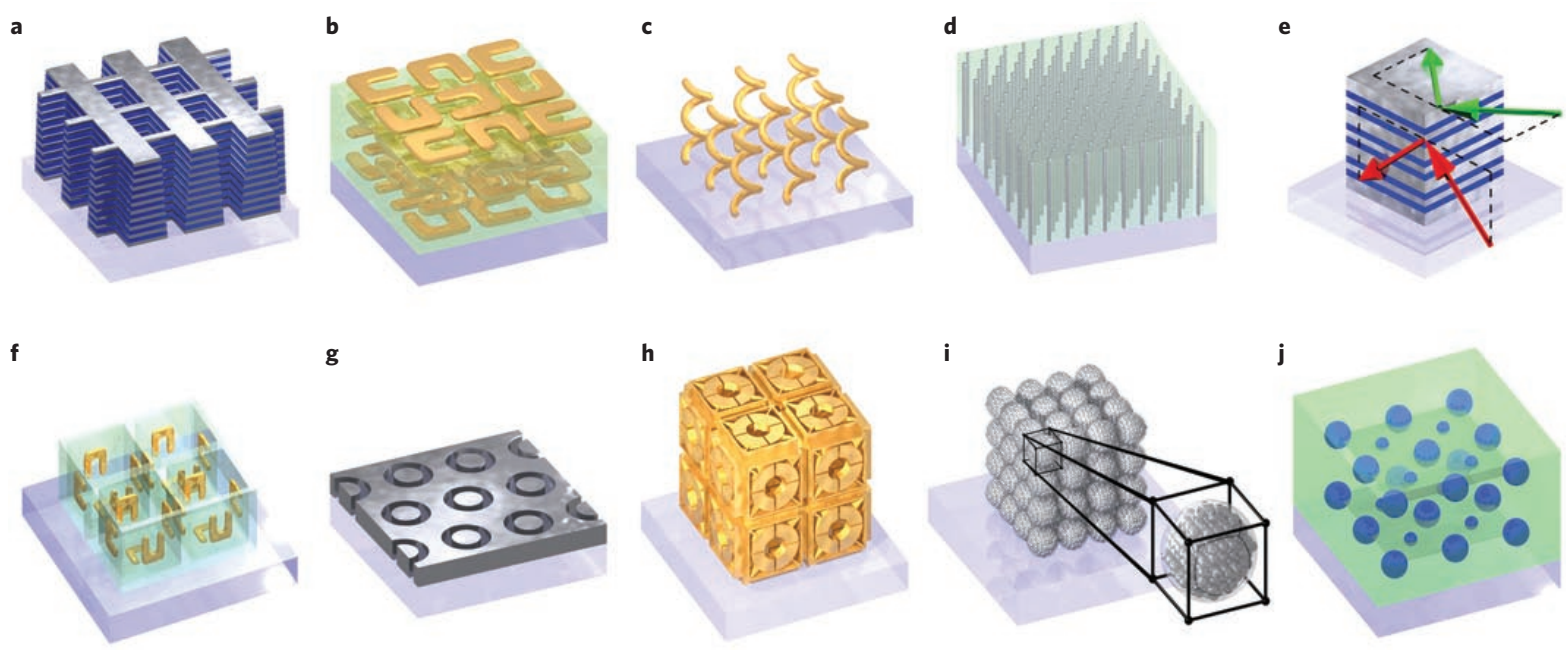

g

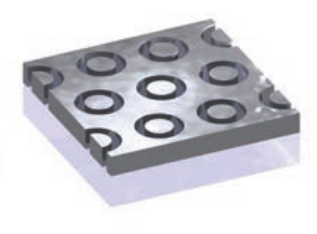

$\mathbf{h}$
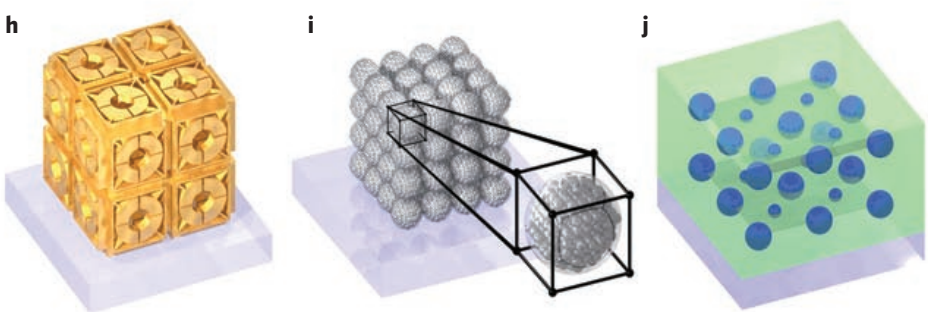

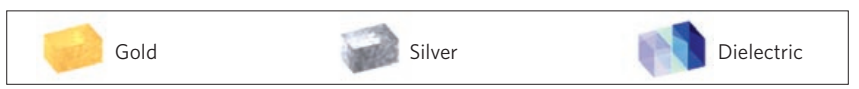

FIG. 1. Examples of metamaterial structures fabricated through composition and periodic repetition of metallic and dielectric elements - 'meta-atoms' or 'metamolecules' (adopted from Ref. [10])

Over the last 10 years, the field of metamaterials has developed as a novel approach for engineering the electromagnetic response of passive micro- and nanostructured materials by engaging resonance excitations, such as localized plasmonic modes and Mie-like resonances. Remarkable results have been achieved including negative-index media that refract light in the opposite direction from that of conventional materials, chiral materials that rotate the polarization state of light hundreds of thousands of times more strongly than natural optical crystals, and structured thin films with remarkably strong dispersion that can slow light. A list of only few such remarkable highlights demonstrated with metamaterials during last 10 years includes: optical magnetism, negative index of refraction, invisibility cloaking, optical circuit components, improved imaging. A few example of nanostructured metamaterials are presented in Fig. 2.
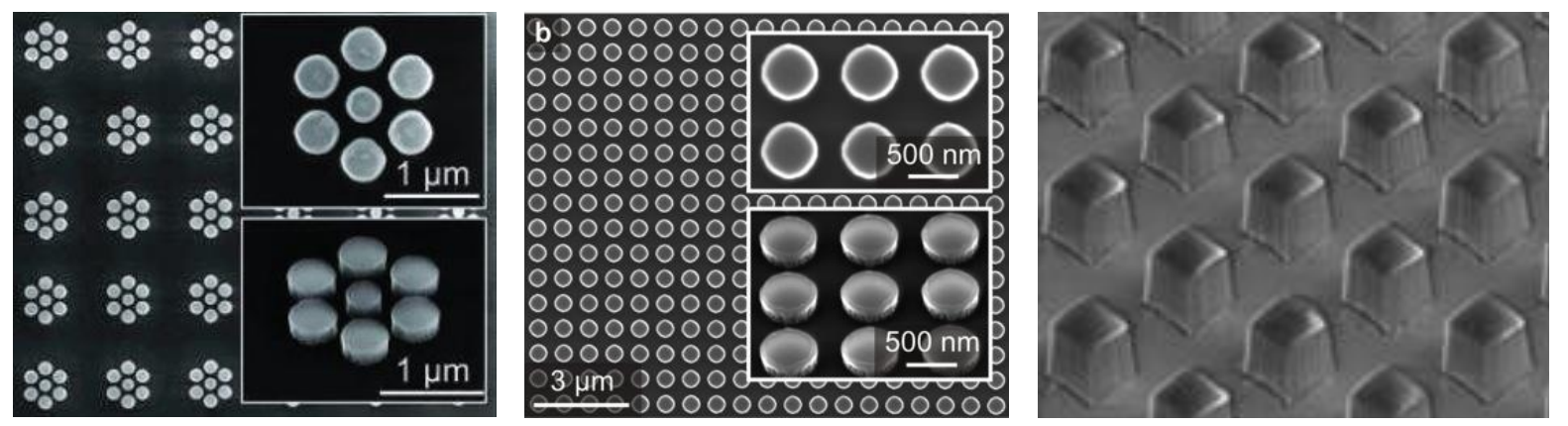

FIG. 2. Examples of metasurfaces with optically resonant electric and magnetic response: nanoantenna oligomers, regular arrays of silicon nanoparticles, and magnetic metamaterial mirrors 


\section{Nonlinear and magnetoelastic metamaterials}

The physics of nonlinear metamaterials originates from an idea to design subwavelength structures having any desired nonlinear response properties [11-13]. Starting from the initial theoretical proposal for the enhancement of nonlinear properties of metamaterials, many nonlinear phenomena with metamaterials were investigated, both theoretically and experimentally. Recent studies cover several topics of magnetoelastic and tunable nonlinear metamaterials for $\mathrm{THz}$ and microwave applications.

As emphasized above, the advent of metamaterials provides novel methodologies for controlling light-matter interaction. With proper design and fabrication of the metamaterial units, - the so-called meta-atoms and meta-molecules, exotic properties unavailable with natural materials can be realized. These studies have inspired many unconventional ideas in optics and photonics, promising various ultra-compact and highly efficient metadevices [14]. Today, an important direction in the development of metadevices is achieving dynamic control over their exotic properties. This can be done by including nonlinear or tunable elements into passive metamaterials, such as liquid crystals; alternatively, one can design meta-molecules with deformable structures. For example, mechanical tuning is now widely used in THz metamaterials. We have recently proposed a post-processing approach for mechanical tuning of the electromagnetic properties of metamaterials, which may be used in applications which require precise engineering of metamaterial resonances.

Structural tuning shows advantages in modulation contrast and the ability to manipulate meta-molecules individually. However, deformation based on static electric or magnetic forces is not so straightforward for optical metamaterials with sub-micrometer features. To solve this problem, our research team introduced the concept of magnetoelastic metamaterials [13], where the meta-molecules can be deformed by the resonance-enhanced electromagnetic force. Importantly, this is a scalable solution of 'light controls light' concept over a wide frequency range, and the interaction between electromagnetic resonance and structural dynamics can demonstrate novel effects beyond a simple modulation of parameters.

To achieve strong electromagnetic-to-elastic interaction, we have introduced an optimized design by exploiting the torsional deformation of chiral meta-molecules. We have demonstrated giant bistable effect and dynamic nonlinear optical activity due to a self-oscillation effect. An important breakthrough we made recently is the prediction and observation of a spontaneous chiral symmetry breaking effect (see Fig. 3) in a metamaterial composed of enantiomeric torsional meta-molecules, i.e. chiral meta-molecules with opposite handedness In such a scenario, the initial configuration of the system is achiral due to chiral symmetry. However, due to the intermolecular electromagnetic interaction, the system stability changes, and such symmetry can be broken spontaneously when the incident power exceeds a certain threshold value, which further leads to an abrupt nonlinear polarization change and mode splitting. The predicted effect is an artificial achiral-chiral phase transition and it was successfully demonstrated in a microwave experiment. As shown in the figure, the single resonance under chiral symmetry configuration splits into two new resonances in the power regime of broken symmetry.

Our studies provide a novel paradigm to achieve artificial nonlinear and phase transition effects in metamaterials via electromagnetic-elastic coupling. The realization of these effects at infrared and optical frequencies can greatly enrich the functionalities of future meta-devices. 
a

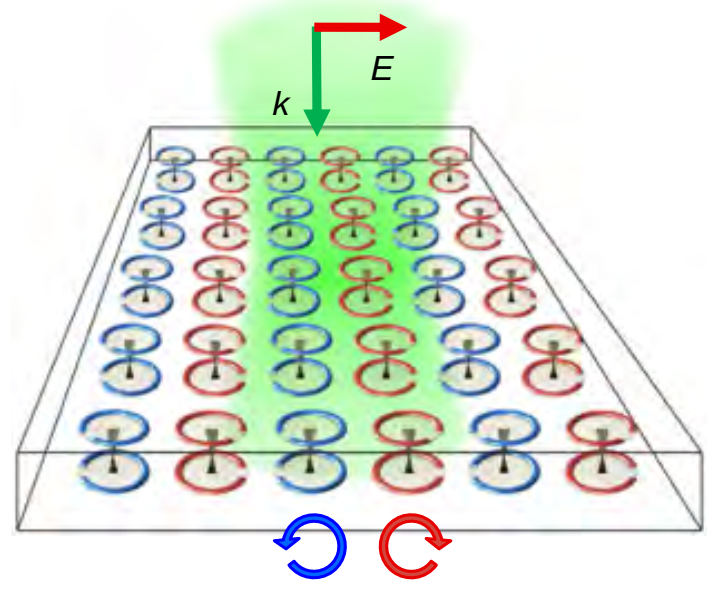

C

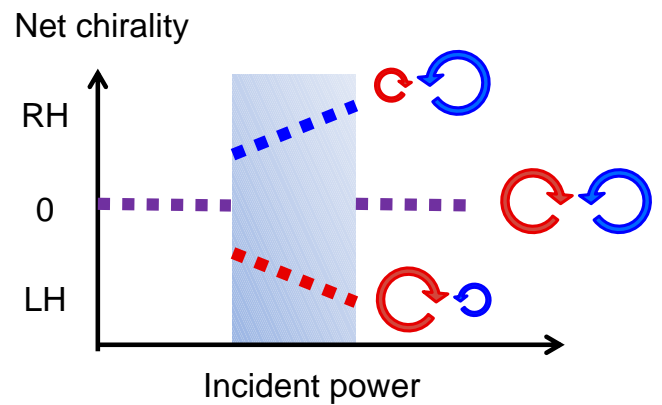

b

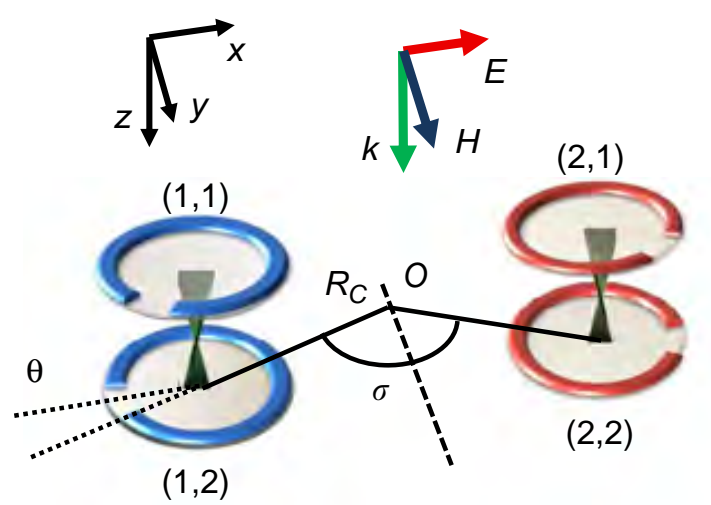

Top view
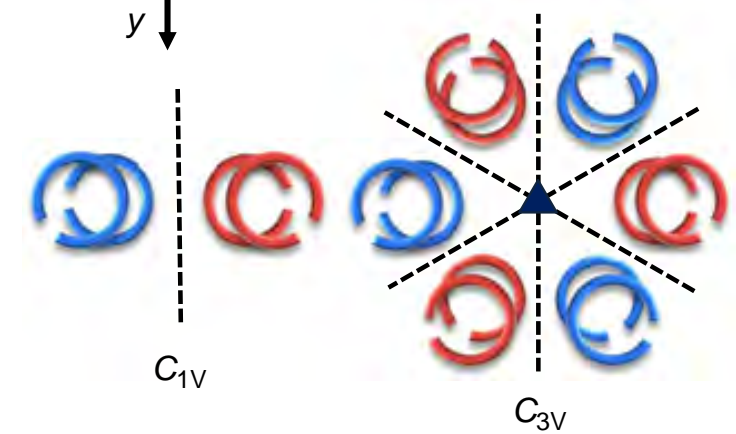

FIG. 3. Schematics of torsional metamaterial enantiomers and spontaneous chiral symmetry breaking [14]. (a) Conceptual layout of torsional metamaterials with chiral symmetry. Each meta-molecule consists of two coaxial twisted meta-atoms connected elastically, and meta-molecules with right and left handedness are plotted in blue and red colours, respectively. The green-shaded region represents the incident electromagnetic waves, and the responses of enantiomers are denoted by circular arrows. (b) Configurations of enantiomeric meta-molecules. Shown are the displacement vector between a meta-molecule and the rotation centre $\mathrm{O}$ and the rotation angle. The dashed lines are the axes of mirror symmetry. (c) Schematic showing the phase transition of net chirality of the system when the incident power changes. In the shaded regime, spontaneous chiral symmetry breaking occurs and the net chirality can fall into either the left-handed or the right-handed regime [15].

\section{Metasurfaces and graphene physics}

Optical metasurfaces are thin-layer subwavelength patterned structures which interact strongly with light, and they became a logical extension of the metamaterials concept with regards to its practical applications. In addition, we now observe the emergence of a new paradigm of metadevices, defined as devices having unique and useful functionalities realized by structuring the functional matter on the subwavelength scale.

One of the important directions in the physics of metasurfaces is the use of graphene as a component of metadevices. Graphene is a two-dimensional array of carbon atoms arranged in a honeycomb lattice, which has remarkable electronic and optical properties. Recently, its 
unique optical properties have generated significant interest in the research community, revealing its great potential for applications in photonics and optoelectronics. The optical response of graphene is characterized by a surface conductivity which is determined by the chemical potential. For photon energies below a certain level, defined by a chemical potential, graphene exhibits a metal-like conductivity. In this regime, similar to metals, graphene can support transverse magnetic (TM) electromagnetic surface plasmon polaritons, and they represent coupled state of the electromagnetic field and electrons. For the range of frequencies above the chemical potential, graphene has dielectric characteristics and it supports transverse electric (TE) surface waves. The study of plasmonic effects in graphene structures has attracted a special interest from the nanoplasmonics research community due to novel functionalities delivered by such systems, including the strong confinement of electromagnetic waves by a graphene layer and tunability of graphene properties through doping or electrostatic gating. Currently, graphene plasmonics is a rapidly growing new field of physics which utilizes concepts of conventional metal plasmonics combined with the unique electronic and optical properties of graphene. Being guided by a graphene monolayer, TM-polarized plasmons with subwavelength localization have an extremely short wavelength, and that is why their excitation is rather challenging. Nevertheless, recent experiments have provided evidence for the existence of such plasmons by means of the scattering near-field microscopy and the nanoimaging.

More recent studies include the analysis of both linear and nonlinear effects in graphene structures, including the nonlinear propagation and switching of light in two coupled layers of graphene (see Fig. 3(a)), demonstrating that this simple double-layer structure can operate as an efficient optical coupler for both continuous plasmon polaritons and for subwavelength spatial solitons. Multi-layer graphene structures were shown to be capable of increasing the plasmon wavelength, thus increasing plasmon propagation length (see Figs. 4(b,c)) and also predicting the existence of plasmon solitons in graphene (see Figs. 4(d-g)).

\section{Towards metadevices}

The ever-increasing demand for faster information transfer and processing drives efforts to remove the bottleneck between fiber-based optical telecommunication networks and electronic data handling and routing, improving data storage and developing parallel data processing operating in a compact space. Fulfilment of these tasks will require strong and fast nonlinearities for switching between different lights, and much improved control of the electromagnetic properties of matter with external stimuli, such as electric signals. Many of these functionalities may be greatly enhanced by hybridizing functional matter with metamaterials and graphene, by exploiting the nonlinearity of the metamaterial framework itself, and by taking advantage of the changing balance of forces in systems with building blocks smaller than the wavelength of light. This leads to the concept of metadevices, a logical extension of the metamaterial paradigm, where interactions are nonlinear and responses are dynamic. We envision that a future platform for highly integrated electromagnetic signal processing and distribution will emerge which will combine nonlinear, memory and switchable functionalities with transformation optics' ability to guide light via the engineered electromagnetic space, using metamaterials with spatially variable parameters. 
(a)
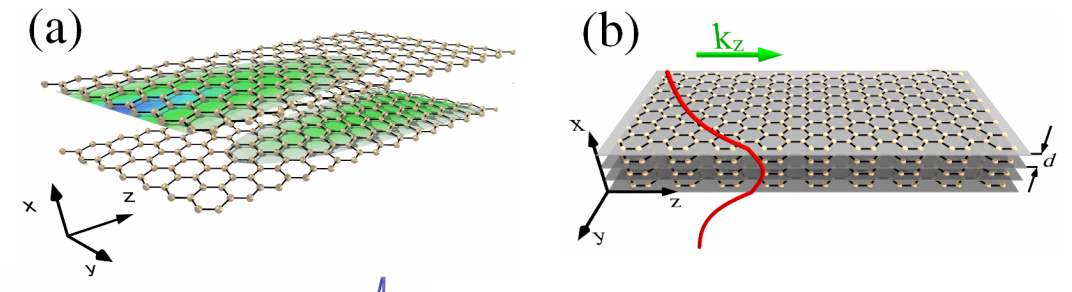

(c)

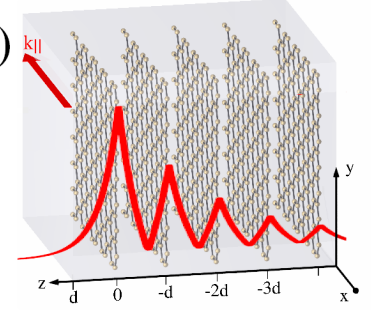

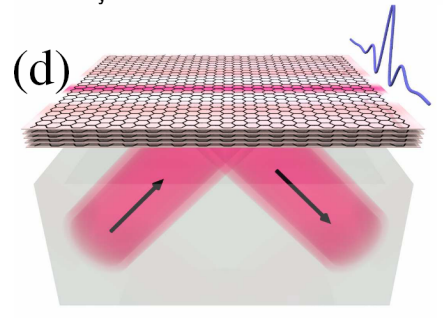
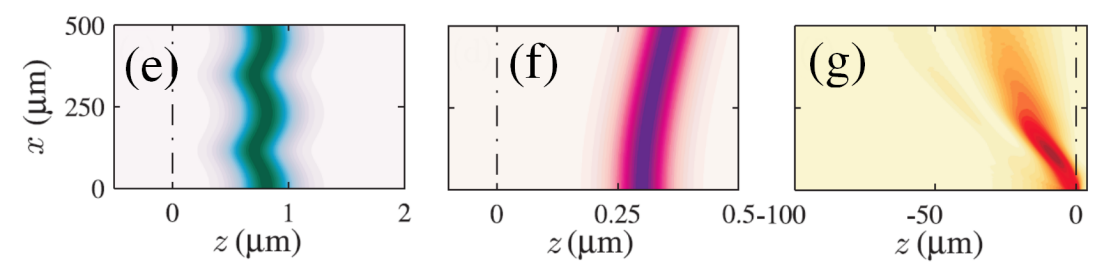

FIG. 4. Examples of linear and nonlinear waveguiding graphene structures [16, 17]: (a) Nonlinear graphene coupler composed of two layers of graphene where light can tunnel from one layer to the other; (b) Multilayer graphene waveguide supporting different types of TM and TE polarized surfaces waves-plasmon polaritons; (c) Optical Tamm states localized the surface of multilayer graphene stack; (d) Schematics of the excitation of a dissipative plasmon soliton by an incoming laser light in a multi-layer graphene waveguide; (e-g) various regimes of the soliton propagation along a interface separating linear and nonlinear dielectric materials covered with a graphene layer: wobbling, deflection, and diffraction, respectively.

\section{Summary and outlook}

Future technologies will demand a huge increase in photonic integration and energy efficiency, far surpassing that of bulk optical components and silicon photonics. Such an integration can be achieved by embedding the data-processing and waveguiding functionalities at the material's level, creating a new paradigm of metadevices. It is now believed that robust and reliable metadevices will allow photonics to compete with electronics not only in telecommunication systems, but also at the level of consumer products such as mobile phones or automobiles. The main challenges in achieving this vision will be in developing cost-efficient fabrication and device integration technologies.

\section{References}

[1] V.G. Veselago. The electrodynamics of substances with simultaneously negative values of $\epsilon$ and $\mu$. Sov. Phys. Usp., 1968, 10, P. 509.

[2] R.A. Shelby, D.R. Smith, S. Schultz. Experimental Verification of a Negative Index of Refraction. Science, 2001, 292, P. 77.

[3] D.R. Smith, W. J. Padilla, et al. Composite medium with simultaneously negative permeability and permittivity. Phys. Rev. Lett., 2000, 84, P. 4184.

[4] J.B. Pendry. Negative Refraction Makes a Perfect Lens. Phys. Rev. Lett., 2000, 85, P. 3966.

[5] D.R. Smith, J.B. Pendry. Reversing Light with Negative Refraction. Phys. Today, 2004, 57 (6), P. 37.

[6] V.M. Shalaev. Optical negative-index metamaterials. Nature Photonics, 2007, 1, P. 41-48.

[7] S. Linden, C. Enkrich, et al. Magnetic response of metamaterials at 100 terahertz. Science, 2004, 306, P. 1351.

[8] T.J. Yen, W.J. Padilla, et al. Terahertz magnetic response from artificial materials. Science, 2004, 303, P. 1494.

[9] A.N. Grigorenko, A.K. Geim, et al. Nanofabricated media with negative permeability at visible frequencies. Nature, 2005, 438, P. 335. 
[10] C. Soukoulis, M. Wegener. Past achievements and future challenges in the development of three-dimensional photonic metamaterials. Nature Photonics, 2011, 5, P. 523.

[11] A.A. Zharov, I.V. Shadrivov, Yu.S. Kivshar. Nonlinear properties of left-handed metamaterials. Phys. Rev. Lett., 2003, 91, P. 037401.

[12] M. Lapine, I.V. Shadrivov, Yu.S. Kivshar. Nonlinear metamaterials. Rev. Mod. Phys., 2014, 86, P. 1093.

[13] I.V. Shadrivov, M. Lapine, Yu.S. Kivshar (Eds). Nonlinear, Active, and Tunable Metamaterials. SpringerVerlag, Berlin, 2015, 324 p.

[14] N.I. Zheludev, Yu.S. Kivshar.From metamaterials to metadevices. Nature Materials, 2012, 11, P. 917.

[15] M. Liu, D.A. Powell, et al. Spontaneous chiral symmetry breaking in metamaterials. Nature Communications, 2014, 5, P. 4441.

[16] D.A. Smirnova, A.V. Gorbach, et al. Nonlinear switching with a graphene coupler. Phys. Rev. B, 2013, 88, P. 045443.

[17] D.A. Smirnova, I.V. Shadrivov, A.I. Smirnov, Yu.S. Kivshar. Dissipative plasmon-solitons in multilayer graphene. Laser Photonics Rev., 2014, 8, P. 291-296. 